\title{
INFERRING GENOME-WIDE MOSAIC STRUCTURE
}

\author{
QI ZHANG, WEI WANG, LEONARD MCMILLAN, \\ FERNANDO PARDO-MANUEL DE VILLENA, DAVID THREADGILL \\ University of North Carolina at Chapel Hill
}

\begin{abstract}
Genetic recombination plays two essential biological roles. It ensures the fidelity of the transmission of genetic information from one generation to the next and it generates new combinations of genetic variants. Therefore, recombination is a critical process in shaping arrangement of polymorphisms within populations. "Recombination breakpoints" in a given set of genomes from individuals in a population divide the genome into haplotype blocks, resulting in a mosaic structure on the genome. In this paper, we study the Minimum Mosaic Problem: given a set of genome sequences from individuals within a population, compute a mosaic structure containing the minimum number of breakpoints. This mosaic structure provides a good estimation of the minimum number of recombination events (and their location) required to generate the existing haplotypes in the population. We solve this problem by finding the shortest path in a directed graph. Our algorithm's efficiency permits genome-wide analysis.
\end{abstract}

\section{Introduction}

Ancestral genetic recombination events play a critical role in shaping extant genomes. Characterizing the patterns of recombination (e.g. the recombination locations and rates), is a crucial step for reconstructing evolutionary histories, performing disease association mapping, and solving other population genetics problems.

During meiosis diploid organisms recombine two homologous genome copies on a chromosome by chromosome basis to form a haploid gamete, which contributes half of the genetic content to its offspring. This mixing of genomes leads to mosaic chromosome (haplotype) structure composed of segments from each grandparent. We refer to the boundaries between the segments of each haplotype as the recombination breakpoints in this paper. Recombination breakpoints represent locations where the crossovers have occurred, either during the generation of the haplotype itself, or in previous generations.

In this paper, we are interested in inferring the possible mosaic structure 
of a given set of related haplotypes. This is accomplished by finding a set of recombination breakpoints that divide the haplotypes into compatible blocks according to the Four-Gamete Test (FGT)? The FGT states that, under the infinite-sites assumption?, all pairs of polymorphisms should cooccur in only three out of their four possible configurations. Thus, when four configurations are observed in a pair of markers, it implies that either a recombination or a homoplastic event has occurred between them. We propose an efficient algorithm to solve the "Minimum Mosaic Problem", which finds the mosaic with the minimum number of breakpoints. The algorithm is suitable for genome-wide study.

\section{Related Work}

Many algorithms have been developed for estimating a lower bound on the minimum number of recombination events necessary to generate a given set of haplotypes. Hudson and Kaplan? proposed a lower bound (HK bound) computed using the FGT? ? Their algorithm computes a minimum set of non-overlapping intervals where all pairs of SNPs in an interval are compatible according to the FGT. This number of intervals, less one, is the HK bound. Myers and Griffiths? proposed a tighter bound, RecMin. However, it is only computationally tractable to find the optimal RecMin in relatively small data sets. Myer and Song et al.? proposed a RecMin approximation algorithm known as HapBound using Integer Linear Programming (ILP). They also proposed an algorithm, SHRUB, which finds a plausible evolutional history for the given haplotypes, called an Ancestral Recombination Graph (ARG). The ARG establishes an upper-bound on the minimum number of recombinations. Different from RecMin or SHRUB, our algorithm focuses on the mosaic structure of a set of sample sequences without explicitly computing the evolutionary history, assuming that the genomic structures of the sample sequences are of more interest. However, the breakpoints on the sample sequences may reflect possible recombination events happened in the history.

In addition to estimating lower-bounds on the number of recombinations, algorithms have also been proposed for finding a set of founders which generate a given set of recombinant sequences. Ukkonen first proposed the founder set reconstruction problem ?. A dynamic programming algorithm was developed to compute a minimum number of founders with a given set of sample haplotype sequences, where a segmentation of all the sequences in the sample set can be derived which contains the minimum number of 
segments. Wu and Gusfield ? studied a slightly different problem to compute a set of $K$ founders where a segmentation of the given sequences can be derived with a minimum number of segments. They proposed a polynomial time algorithm for genotype sample sequences with only two founders. Different from these works, we do not construct the founder sequences, or rely on the existence of a founder set for segmentation and inferring the mosaic structures on the genome.

\section{Problem Formulation}

Suppose that we have a set of $n$ haplotypes over $m$ SNPs, represented by a binary data matrix $D=\left[d_{i j}\right]_{i=1 . . n, j=1 . . m}$. Row $i$ in $D$ corresponds to a haplotype $h_{i}$, and column $j$ in $D$ corresponds to a SNP $s_{j}$. Matrix entry $d_{i j}$ is either 0 or 1 , representing the majority allele or minority allele at SNP $s_{j}$ respectively. In this paper, we only consider crossover recombination events, ignoring gene conversion and homoplasy (assuming they do not have a significant role).

Over any pair of SNPs $s_{j}$ and $s_{j^{\prime}}$, a haplotype takes one of four possible gametes $00,01,10,11$ (the combination of alleles at $s_{j}$ and $s_{j^{\prime}}$ ). We denote the set of haplotypes taking 00,01, 10, or 11 at SNP pair $\left(s_{j}, s_{j^{\prime}}\right)$ as HapSet $t_{00}^{j, j^{\prime}}$, HapSet $_{01}^{j, j^{\prime}}$, HapSet $_{10}^{j, j^{\prime}}$, HapSet $_{11}^{j, j^{\prime}}$ respectively. If all four sets are nonempty, according to the $\mathrm{FGT}^{\text {? }}$, a historical recombination event must have occurred between $s_{j}$ and $s_{j^{\prime}}$. In this case, we say that the SNP pair $\left(s_{j}, s_{j^{\prime}}\right)$ is incompatible. We represent a recombination breakpoint as a tuple $\left(h_{i}, s_{b}\right)$, where the breakpoint locates on haplotype $h_{i}$ between SNPs $s_{b}$ and $s_{b+1}$. It is possible that multiple haplotypes may have breakpoints at the same location since they may "inherit" the breakpoint from a common ancestor sequence.

We define a compatible block of SNPs as a continuous set of SNPs such that any two SNPs inside the block are compatible. Two SNP blocks are incompatible with each other if there exist two incompatible SNPs, one from each block.

A complete set of breakpoints creates a haplotype mosaic structure over the set of genome sequences. A Mosaic $M$ over a SNP data matrix $D$ is defined as a set of recombination breakpoints $M=\left\{\left(h_{i}, s_{b}\right)\right\}, i \in[1, n], b \in$ $[1, m]$. The set of distinct ${ }^{\text {a }}$ locations of breakpoints $s_{b}$ in $M$ divides the entire range of SNPs $\left[s_{1}, s_{m}\right]$ into blocks that satisfy: 1) each block is a

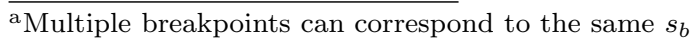


compatible block, 2) any two neighboring blocks are incompatible, and 3) any two neighboring blocks (assume the boundary is between $s_{b}$ and $s_{b+1}$ ) would become compatible if the set of haplotypes that have breakpoints between $s_{b}$ and $s_{b+1}$ are excluded. In this paper, we develop an efficient algorithm for computing Minimum Mosaic (denoted as $M_{\text {min }}$ ) - the mosaic structure that contains the least number of breakpoints. We refer to this problem as the Minimum Mosaic Problem.

\section{Inferring the Local Mosaic}

\subsection{Maximal Intervals}

We begin by defining the concept of maximal interval. An interval $I=$ $\left[s_{b}, s_{e}\right]$ is a set of consecutive SNPs which are compatible with each other starting from $s_{b}$ and ending at $s_{e}$. We define an interval $I$ as a maximal interval if and only if there is no other interval $I^{\prime}, I^{\prime} \neq I, I^{\prime}=\left[s_{b}^{\prime}, s_{e}^{\prime}\right]$, which contains $I: s_{b}^{\prime} \leq s_{b}$, and $s_{e}^{\prime} \geq s_{e}$. The complete set of maximal intervals can be computed in $O(m n)$ time $^{\text {? }}$, assuming that the compatibility test of any two SNPs using FGT takes $O(1)$ time?

\subsection{Finding Local Breakpoints}

Maximal intervals are useful for inferring the local mosaic. The set of distinct breakpoint locations $s_{b}$ in a mosaic $M=\left\{\left(h_{i}, s_{b}\right)\right\}$ divide the entire SNP range $\left[s_{1}, s_{m}\right]$ into compatible blocks, where neighboring blocks are incompatible. The set of breakpoints in $M$ is the union of the set of breakpoints on the boundary of each pair of neighboring blocks. We first consider the breakpoints on the boundary of a pair of neighboring blocks. We observe that, every pair of neighboring blocks in $M$ fall inside a pair of overlapping or adjacent maximal intervals, as stated in the following Lemma:

Lemma 4.1. For any pair of neighboring blocks $\left(B_{L}, B_{R}\right)$ deduced by a mosaic, there exists a pair of overlapping or adjacent maximal intervals $\left(I_{L}, I_{R}\right)$, where $B_{L}$ completely falls inside $I_{L}\left(B_{L} \subseteq I_{L}\right)$ but not $I_{R}$ $\left(B_{L} \backslash I_{R} \neq \phi\right)$, and $B_{R}$ completely falls inside $I_{R}\left(B_{R} \subseteq I_{R}\right)$ but not $I_{L}$ $\left(B_{R} \backslash I_{L} \neq \phi\right)$. We refer to $\left(I_{L}, I_{R}\right)$ as $\left(B_{L}, B_{R}\right)$ 's containing interval pair; and $\left(B_{L}, B_{R}\right)$ as $\left(I_{L}, I_{R}\right)$ 's contained block pair (Fig. 1).

\section{Proof.}




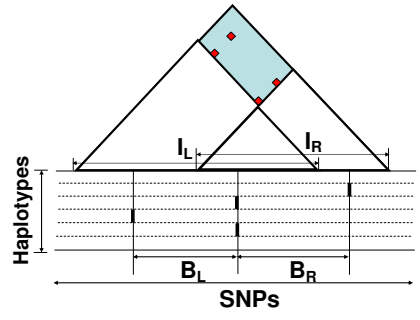

(a) $I_{L}$ and $I_{R}$ are overlapping

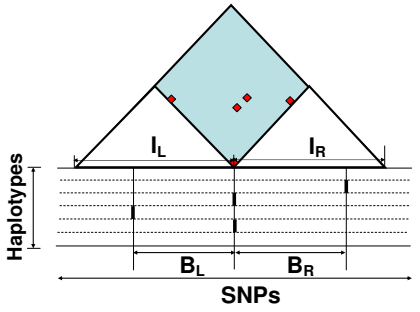

(b) $I_{L}$ and $I_{R}$ are adjacent

Figure 1. Neighboring blocks $B_{L}, B_{R}$ fall inside overlapping/adjacent maximal intervals $I_{L}, I_{R}$ respectively. The dots in the shaded region represent incompatible SNP pairs of $I_{L}$ and $I_{R}$.

Details of the proof are presented in ?

For each pair of overlapping or adjacent intervals $\left(I_{L}, I_{R}\right)$, there exists a set of incompatible SNP pairs SNPPair $\left(I_{L}, I_{R}\right)=\left\{\left(s_{l}, s_{r}\right)\right\}$, where $l<r, s_{l}$ and $s_{r}$ are incompatible, and $s_{l} \in I_{L} \backslash I_{R}, s_{r} \in I_{R} \backslash I_{L}$. For example, in Fig. 1(a) and 1(b), each dot represents an incompatible SNP pair. Let $\left(B_{L}, B_{R}\right)$ be a contained block pair of the interval pair $\left(I_{L}, I_{R}\right)$. We denote the incompatible SNP pairs contained in $\left(B_{L}, B_{R}\right)$ as $\operatorname{SNPPair}\left(B_{L}, B_{R}\right)=\left\{\left(s_{l}, s_{r}\right)\right\}$, where $l<r, s_{l}$ and $s_{r}$ are incompatible, and $s_{l}, s_{r} \in B_{L} \cup B_{R}$. Apparently, SNPPair $\left(B_{L}, B_{R}\right)$ is a subset of SNPPair $\left(I_{L}, I_{R}\right)$. The incompatible SNP pairs in $S N P P a i r\left(B_{L}, B_{R}\right)$ determine the minimum number of the breakpoints on the boundary of $B_{L}$ and $B_{R}$, as well as the corresponding set of haplotypes having these breakpoints. Given an interval pair, several candidate block pairs may be derived, each of which corresponds to a different SNPPair $\left(B_{L}, B_{R}\right)$. Fig. 2(a)-2(d) show four different candidate block pairs derived from the same interval pair. The exact set of incompatible SNP pairs in SNPPair $\left(B_{L}, B_{R}\right)$ depends on the positions of $B_{L}$ and $B_{R}$, i.e., the leftmost SNP of $B_{L}$, and the rightmost SNP of $B_{R}$. Formally, we define the start range $R_{s}$, the end range $R_{e}$ of a block pair $\left(B_{L}, B_{R}\right)$ as the ranges where SNPPair $\left(B_{L}, B_{R}\right)$ remains unchanged if the leftmost SNP of $B_{L}$ changes within $R_{s}$ and the rightmost SNP of $B_{R}$ changes within $R_{e}$. Moreover, the breakpoint range $R_{b}$ of $\left(B_{L}, B_{R}\right)$ is defined as the range where the boundary of $B_{L}$ and $B_{R}$ falls into. The breakpoint range is the overlapping region of $I_{L}$ and $I_{R}$ (if $I_{L}$ and $I_{R}$ overlap), or the boundary of $I_{L}$ and $I_{R}$ (if $I_{L}$ and $I_{R}$ are adjacent to each other). For example, in Fig. 2(a), $\operatorname{SNPPair}\left(B_{L}, B_{R}\right)$ contains only one incompatible SNP pair $\left(s_{q}, s_{r}\right)$. The start range $R_{s}\left(B_{L}, B_{R}\right)$ is $\left(s_{p}, s_{q}\right]$, the end range $R_{e}\left(B_{L}, B_{R}\right)$ is $\left[s_{r}, s_{s}\right)$, and 
the breakpoint range $R_{b}\left(B_{L}, B_{R}\right)$ is $I_{L} \cap I_{R}$. In Fig. 2(b), $S N P P a i r\left(B_{L}, B_{R}\right)$ contains two incompatible SNP pairs $\left(s_{q}, s_{r}\right)$ and $\left(s_{p}, s_{r}\right)$. The start range $R_{s}\left(B_{L}, B_{R}\right)$ is $\left[s^{*}, s_{p}\right]\left(s^{*}\right.$ denotes the leftmost SNP of interval $\left.I_{L}\right)$, and the end range $R_{e}\left(B_{L}, B_{R}\right)$ is $\left[s_{r}, s_{s}\right)$, and the breakpoint range $R_{b}\left(B_{L}, B_{R}\right)$ is $I_{L} \cap I_{R}$.

Any contained block pair $\left(B_{L}, B_{R}\right)$ of any overlapping/adjacent maximal interval pair $\left(I_{L}, I_{R}\right)$ can be a possible neighboring block pair inside a mosaic $M$. A subset of these block pairs constitute a mosaic. Specifically, for any neighboring block pair $\left(B_{L}, B_{R}\right)$ which is inside a Minimum Mosaic $M_{\text {min }}$, we have the following Lemma:

Lemma 4.2. Let $\left(B_{L}, B_{R}\right)$ be a neighboring block pair in a Minimum Mosaic $M_{\text {min }}$, and Breakpoints $\left(B_{L}, B_{R}\right)$ be the set of breakpoints on the boundary of $B_{L}$ and $B_{R}$ in $M_{\text {min }}$, and HapSet (Breakpoints $\left.\left(B_{L}, B_{R}\right)\right)$ be the set of haplotypes having breakpoints in Breakpoints $\left(B_{L}, B_{R}\right)$. Then Breakpoints $\left(B_{L}, B_{R}\right)$ is the smallest number of breakpoints which satisfies:

$$
\begin{aligned}
& \forall\left(s_{l}, s_{r}\right) \in S N P P a i r\left(B_{L}, B_{R}\right) \\
& \left(\exists g_{l, r} \in\{00,01,10,11\}, \text { HapSet }\left(\text { Breakpoints }\left(B_{L}, B_{R}\right)\right) \supseteq \text { HapSet }_{g_{l, r}}^{l, r}\right)
\end{aligned}
$$

Proof. Details of the proof are presented in ?

It is easy to compute Breakpoints $\left(B_{L}, B_{R}\right)$ if $\operatorname{SNPPair}\left(B_{L}, B_{R}\right)$ only contains one pair of incompatible SNPs (as shown in Fig. 2(a)). We can choose the smallest set of HapSet ${ }_{00}^{l, r}$, HapSet ${ }_{01}^{l, r}$, HapSet ${ }_{10}^{l, r}$ and HapSet $t_{11}^{l, r}$ to be Breakpoints $\left(B_{L}, B_{R}\right)$. If $S N P P a i r\left(B_{L}, B_{R}\right)$ contains more than one pair of incompatible SNPs (as shown in Fig. 2(b), 2(c), and 2(d)), we need to find the smallest set of haplotypes which is a superset of at least one of HapSet ${ }_{00}^{l, r}$, HapSet $_{01}^{l, r}$, HapSet $_{10}^{l, r}$ and HapSet ${ }_{11}^{l, r}$, for each pair of incompatible SNPs $\left(s_{l}, s_{r}\right)$. The computation complexity is $O\left(4^{k}\right)$, where $k=\mid$ Breakpoints $\left(B_{L}, B_{R}\right) \mid$. In practice, $k$ is small. Moreover, many incompatible SNP pairs are caused by a small number of SNP patterns, which enables further reduction in computation.

\section{Finding Minimum Mosaic - A Graph Problem}

The set of all possible block pairs $\left\{\left(B_{L}, B_{R}\right)\right\}$ of all overlapping/adjacent maximal interval pairs are the building blocks of a mosaic. We can use them and construct a graph as follows. A node $n d$ in this graph represents the 


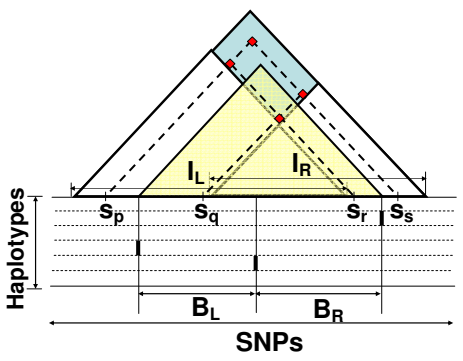

(a)

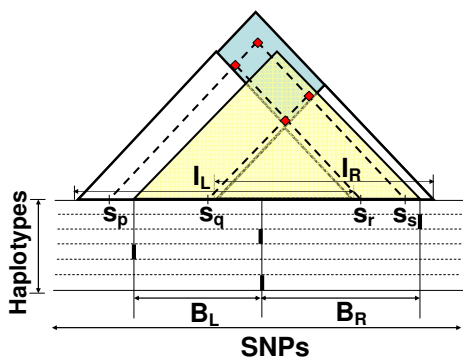

(c)

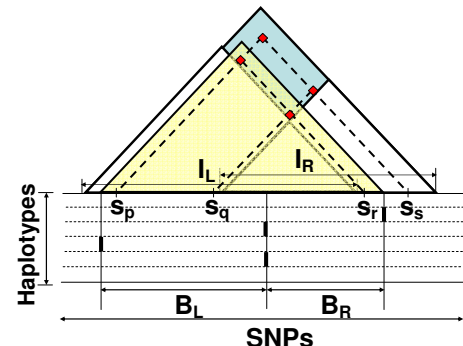

(b)

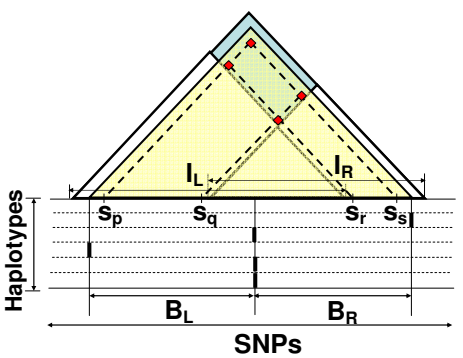

(d)

Figure 2. Neighboring blocks $B_{L}, B_{R}$ contain different subsets of the incompatible SNP pairs. The dots represent the incompatible SNP pairs contained in the overlapping maximal intervals $I_{L}$ and $I_{R}$. The dots inside the shaded triangle are contained in the neighboring block pair $B_{L}$ and $B_{R}$.

combination of three block pairs $B P_{1}=\left(B_{L_{1}}, B_{R_{1}}\right), B P_{2}=\left(B_{L_{2}}, B_{R_{2}}\right)$, $B P_{3}=\left(B_{L_{3}}, B_{R_{3}}\right)$ that satisfy the following constraints: 1$)$ the breakpoint range of $B P_{1}$ overlaps with the start range of $B P_{2}: R_{b}\left(B P_{1}\right) \cap R_{s}\left(B P_{2}\right) \neq \phi$; 2) the end range of $B P_{1}$, the breakpoint range of $B P_{2}$, and the start range of $B P_{3}$ overlap: $\left.R_{e}\left(B P_{1}\right) \cap R_{b}\left(B P_{2}\right) \cap R_{s}\left(B P_{3}\right) \neq \phi ; 3\right)$ the end range of $B P_{2}$ overlaps with the breakpoint range of $B P_{3}: R_{e}\left(B P_{2}\right) \cap R_{b}\left(B P_{3}\right) \neq \phi$. As shown in Fig. $3, B P_{1}, B P_{2}$, and $B P_{3}$ are the left block pair, middle block pair, and right block pair of $n d$, respectively. The breakpoint range of $n d$ is the intersection of the end range of $B P_{1}$, the breakpoint range of $B P_{2}$, and the start range of $B P_{3}: R_{b}(n d)=R_{e}\left(B P_{1}\right) \cap R_{b}\left(B P_{2}\right) \cap R_{s}\left(B P_{3}\right)$. The set of breakpoints associated with $n d$ is the same as Breakpoints $\left(B P_{2}\right)$, denoted as Breakpoints $(n d)$. The weight of the node is the number of breakpoints in Breakpoints(nd), weight $(n d)=\mid$ Breakpoints $(n d) \mid$.

We also create two special kinds of nodes - starting nodes and ending nodes to model the two ends of a chromosome. We first identify all 


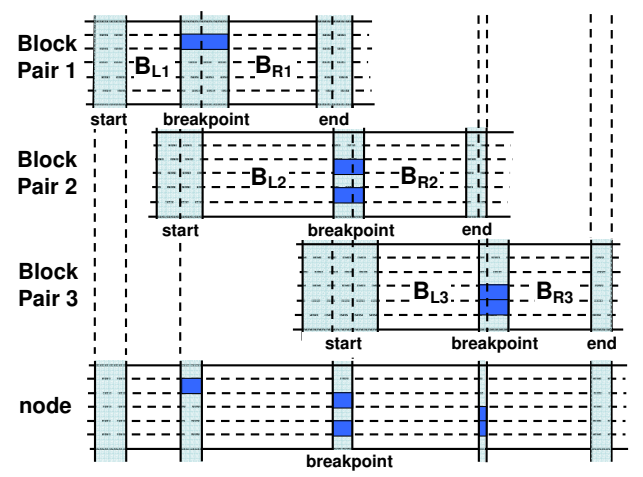

Figure 3. Three block pairs form a node. Block pair 1, 2, and 3 are the left, middle, and right block pair of the node respectively. The breakpoint range of the node is the intersection of the end range of block pair 1, the breakpoint range of block pair 2, and the start range of block pair 3. The vertical stripes correspond to the start range, breakpoint range, and end range of a block. The marked haplotypes in the stripes are the haplotypes which have breakpoints in the corresponding region.

block pairs with start range beginning from the first SNP $s_{1}$, referred to as starting block pairs. We create a starting node $n d_{s}$ for every combination of a starting block pair $B P_{s}$ and another block pair $B P$ satisfying 1) the breakpoint range of $B P_{s}$ overlaps with the start range of $B P$ : $R_{b}\left(B P_{s}\right) \cap R_{s}(B P) \neq \phi$, and 2$)$ the end range of $B P_{s}$ overlaps with the breakpoint range of $B P: R_{e}\left(B P_{s}\right) \cap R_{b}(B P) \neq \phi$. BP $P_{s}$ is the middle block pair of the starting node $n d_{s}, B P$ is the right block pair of $n d_{s}$. There is no left block pair for $n d_{s}$. The set of breakpoints associated with $n d_{s}$ is the same as Breakpoints $\left(B P_{s}\right)$ : Breakpoints $\left(n d_{s}\right)=$ Breakpoints $\left(B P_{s}\right)$. The weight of $n d_{s}$ is weight $\left(n d_{s}\right)=\mid$ Breakpoints $\left(n d_{s}\right) \mid$. Similarly, we create a set of ending nodes $\left\{n d_{e}\right\}$ associated with the set of ending block pairs $\left\{B P_{e}\right\}$.

After generating all nodes, we connect nodes with directed edges according to the following rule. For nodes $n d_{1}$ and $n d_{2}$, if $n d_{1}$ 's middle block pair is the same as $n d_{2}$ 's left block pair and $n d_{1}$ 's right block pair is the same as $n d_{2}$ 's middle block pair, we add an edge from $n d_{1}$ to $n d_{2}$. The nodes and edges form a directed graph. A Minimum Mosaic corresponds to a shortest path from any starting node to any ending node in this graph. The weight of the path is the sum of all node weights on the path. The set of breakpoints $\{$ Breakpoints $(n d)\}$ associated with all nodes on the shortest path is the Minimum Mosaic solution. We can use any shortest path algorithm to compute the solution. The details of the complete algorithm 
and the correctness proof are presented in?

\section{Experimental Studies}

Our algorithm is implemented in $\mathrm{C}++$ and all experiments were performed on a machine with an Intel Core2 Duo processor of 1.60GHz and 3GB RAM.

\subsection{Kreitman's ADH Data}

The alcohol dehydrogenase (ADH) data of Kreitman? consists of 11 haplotypes over 43 polymorphic sites of the ADH locus of fruit fly, Drosophila melanogaster. The haplotypes were sampled from 5 geographically distinct populations: Washington, Florida, Africa, France, and Japan? Our algorithm detected 7 breakpoints shown in Fig. 4(a). We can estimate the exact locations of 6 out of 7 breakpoints: $H_{1}:\left(S_{3}, S_{4}\right), H_{5}:\left(S_{3}, S_{4}\right)$, $H_{5}:\left(S_{16}, S_{17}\right), H_{5}:\left(S_{35}, S_{36}\right), H_{6}:\left(S_{35}, S_{36}\right), H_{6}:\left(S_{36}, S_{37}\right)$. For the remaining breakpoint on $H_{1}$, its location can be either $\left(S_{12}, S_{13}\right)$, or $\left(S_{13}, S_{14}\right)$, or $\left(S_{14}, S_{15}\right)$, or $\left(S_{15}, S_{16}\right)$ with equal probability.

Note that 7 is the lower and upper bounds of the minimum number of recombinations, estimated by HapBound and SHRUB, respectively? ${ }^{\text {. There- }}$ fore, 7 is the exact number of minimum number of recombination events for the ADH data. The corresponding ARG generated by SHRUB is shown in Fig. 4(c). The breakpoints in the ARG are illustrated in a SNP matrix in Fig. 4(b). By comparing Fig. 4(a) and 4(b), we observe that almost the same set of breakpoints are inferred by our algorithm and SHRUB.

\subsection{Running Time and Scalability Analysis}

We tested the performance of our algorithm on two genome-wide SNP data sets from mouse. Both sets represent a combination of experimental and imputed genotypes? in two overlapping sets of laboratory inbred strains available from the Center of Genome Dynamics at the Jackson's Laboratory?. The 51-strain data set contains 51 inbred mouse strains with $7,870,134 \mathrm{SNPs}^{\mathrm{b}}$. The 74 -strain data set contains 74 inbred mouse strains

\footnotetext{
${ }^{\mathrm{b}}$ The 51-strain data set includes Chr 1-19 and Chr X, with 51 mouse strains: X129S1.SvImJ, X129S4.SvJae, X129X1.SvJ A.J, AKR.J, BALB.cByJ, BTBR.T...tf.J, BUB.BnJ, C3H.HeJ, C57BL.6J, C57BLKS.J, C57BR.cdJ, C57L.J, C58.J, CAST.EiJ, CBA.J, CE.J, CZECHII.EiJ, DBA.1J, DBA.2J, DDK.Pas, FVB.NJ, I.LnJ, JF1.Ms, KK.HLJ, LG.J, LP.J, MA.MyJ, MAI.Pas, MOLF.EiJ, MSM.Ms, NOD.LtJ, NON.LtJ, NZB.BINJ, NZO.HILtJ, NZW.LacJ, O20, PERA.EiJ, PL.J, PWD.Ph, PWK.PhJ, Qsi5, RIIIS.J, SEA.GnJ, SEG.Pas, SJL.J, SM.J, SPRET.EiJ, ST.bJ, SWR.J, and WSB.EiJ.
} 
$\begin{array}{lllllllll}\boldsymbol{s}_{1} & \boldsymbol{s}_{5} & \boldsymbol{s}_{10} & \boldsymbol{s}_{15} & \boldsymbol{s}_{20} & \boldsymbol{s}_{25} & \boldsymbol{s}_{30} & \boldsymbol{s}_{35} & \boldsymbol{s}_{43}\end{array}$ $h_{1} 00000001100 \quad 0001101110111100000000000000$ $\boldsymbol{h}_{2}-0010000000000000001101110111100000000000000$ $h_{3}-0000000000000000000000000000000000010000101$ $\boldsymbol{h}_{4}-0000000000000000110000000000000000010011000$ $h_{5}-000 \$ 100010110011100000000000000000$ 1000000 $\boldsymbol{h}_{6}-0010000000000001000000000000001010101000010$ $h_{6}-001000000000010000000000010101+1000010$ $\boldsymbol{h}_{7}-0010000000000001000000000000011111101000000$
$\boldsymbol{h}_{8}-1111100010111001000000000000011111101100000$ $\boldsymbol{h}_{g}-1111100010111001000000000000011111101100000$ $h_{10} 1111100010111001000000000000011111101100000$ $h_{11} 1111111110000101000010001000011111101000000$

(a) $\begin{array}{lllllllll}s_{1} & s_{5} & s_{10} & s_{15} & s_{20} & s_{25} & s_{30} & s_{35} & s_{43}\end{array}$ $h_{1}$ 00010000011000000001101110111100000000000000 $\boldsymbol{h}_{\mathbf{2}}-0010000000000000001101110111100000000000000$ $h_{3}-0000000000000000000000000000000000010000101$ $h_{4}-0000000000000000110000000000000000010011000$ h -000110001011001141000000000000000001000000 $101+1,000010$ $h_{7}-0010000000000001000000000000011111101000000$ $\boldsymbol{h}_{8}-1111100010111001000000000000011111101100000$ $\boldsymbol{h}_{9}-1111100010111001000000000000011111101100000$ $h_{10}-1111100010111001000000000000011111101100000$ $h_{11} 1111111110000101000010001000011111101000000$

(b)

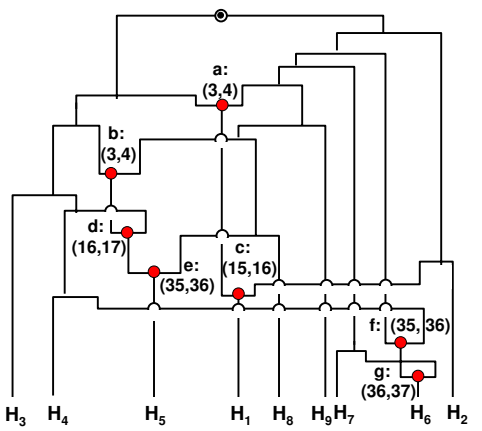

(c)

Figure 4. Comparison of Minimum Mosaic and Hapbound/SHRUB results on ADH data. (a): the Minimum Mosaic result; (b): the result inferred from the ARG in (c); (c): the ARG computed using SHRUB? The bars in (a) and (b) represent the breakpoints. The dots in (c) represents the recombination events.

with $7,989,200 \mathrm{SNPs}^{\mathrm{c}}$.

Fig. 5 shows the running time comparison of Hapbound and our algorithm using the first $w$ SNPs from Chromosome 19 of both data sets where $w$ varies from 1000 to 4000 . Our algorithm is $250 \mathrm{x}-7000 \mathrm{x}$ faster than Hapbound on 74-strain dataset, and 350x - 4000x faster on 51-strain dataset.

Our algorithm is efficient enough to finish on all chromosomes (Chr 119 and Chr X). Results from the 51-strain data set are shown in Table 1. Genome-wide, the number of breakpoints in the Minimum Mosaic varies between 15253 (Chr X) and 266006 (Chr 1), and the number of derived blocks in the Minimum Mosaic varies between 9888 (Chr X) and 68261

${ }^{\mathrm{C}}$ The 74 -strain dataset includes all strains in the 51-strain data set and 23 additional strains: BALB.cJ, BPH.2J, BPL.1J, BPN.3J, C57BL.10J, CALB.RK, DDY.JCLSIDSEYFRKJ, EL.SUZ_2, HTG.GOSFSN, ILS, IS.CAMRK, ISS, LEWES.EI, MOLG.DN, MRL.MpJ, NOR.LTJ, P.J, PERC.EI, RF.J, SKIVE.EI, SOD1.EI, TALLYHO.JNGJ, and ZALENDE.EiJ. 


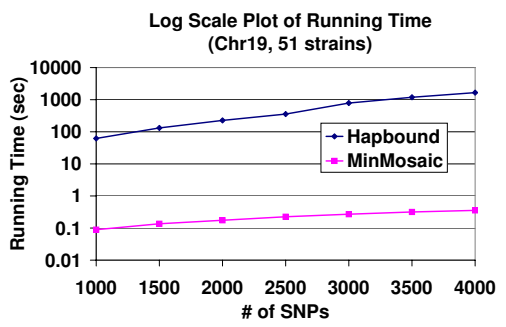

(a) 51-strain Dataset

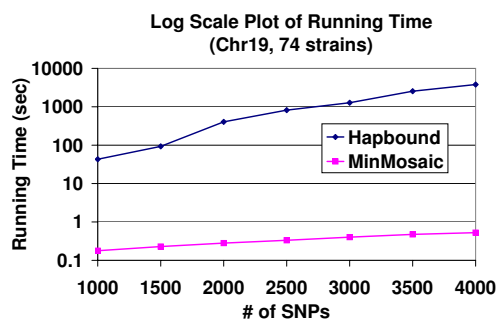

(b) 74-strain Dataset

Figure 5. Comparison of the running times of MinMosaic and Hapbound over varying number of SNPs (in log scale). The datasets used are from Chr19 of 51-strain dataset and 74-strain dataset. The number of SNPs included varies from 1000 to 4000 .

(Chr 1). The average number of breakpoints per neighboring block pair is 2.2 .

Table 1. The result on genome-wide 51-strain mouse data set.

\begin{tabular}{ccccc}
\hline Chr & \# of SNP & \# of breakpoints & \# of blocks & Runtime $(\mathrm{min})$ \\
1 & 694809 & 266006 & 68261 & 6.87 \\
2 & 524667 & 210797 & 47793 & 11.27 \\
3 & 509892 & 113715 & 52487 & 8.90 \\
4 & 476425 & 100702 & 43776 & 7.84 \\
5 & 496888 & 110157 & 49938 & 33.98 \\
6 & 509547 & 97740 & 49562 & 6.42 \\
7 & 405733 & 94973 & 46884 & 38.83 \\
8 & 444910 & 87659 & 45796 & 37.10 \\
9 & 361571 & 86755 & 40189 & 3.89 \\
10 & 399126 & 64806 & 35764 & 3.21 \\
11 & 259028 & 65092 & 27575 & 23.52 \\
12 & 396114 & 89243 & 42159 & 1.30 \\
13 & 399930 & 75323 & 39914 & 3.03 \\
14 & 345783 & 67304 & 34089 & 2.54 \\
15 & 337461 & 78181 & 35776 & 4.08 \\
16 & 305078 & 57257 & 28449 & 1.14 \\
17 & 266421 & 73542 & 31517 & 0.75 \\
18 & 291266 & 69546 & 31271 & 8.61 \\
19 & 222031 & 49276 & 22839 & 1.46 \\
$\mathrm{X}$ & 223456 & 15253 & 9888 & 0.96
\end{tabular}

\section{Conclusions}

Genetic recombination during meiosis generates a mosaic structure of the genome, where each resulting haplotype consists of segments from different ancestral sequences. In this paper, we study the Minimum Mosaic model that contains a minimum number of breakpoints to generate the haplotypes present within extant populations. The resulting blocks are compatible where no recombinations can be inferred within a block according to the 
FGT. We proposed a novel algorithm to compute the minimum mosaic structure of genomes. the efficiency of our algorithm allows for genomewide analysis.

\section{References}

1. Gusfield,D. (2005) Optimal, efficient reconstruction of root-unknown phylogenetic networks with constrained and structured recombination, Journal of Computer and System Sciences, 70, 381-398.

2. Hudson,R., Kaplan,N. (1985) Statistical properties of the number of the recombination events in the history of a sample of DNA sequences, Genetics, 111, 147-164.

3. Kreitman,M. (1983) Nucleotide polymorphism at the alcohol dehydrogenase locus of Drosophila melanogaster, Nature, 304, 412-417.

4. Moore,K., Zhang,Q, McMillan,L., Wang,W., Pardo-Manuel de Villena,F. (2008) Genome-wide compatible SNP intervals and their properties, UNC Tech Report, June 08.

5. Myers,S.R., Griffiths,R.C. (2003) Bounds on the minimum number of recombination events in a sample history, Genetics, 163, 375-394.

6. Schwartz,R., Halldorson,B.V., Bafna,V., Clark,A.G., Istrail,S. (2003) Robustness of inference of haplotyp block structure, J. Comput. Biol., 10, 13-19.

7. Song,Y.S., Wu,Y, Gusfield,D. (2005) Efficient computation of close lower and upper bounds on the minimum number of recombinations in biological sequence evolution, Bioinformatics, 21, i413-i422.

8. Song,Y.S., Hein,J. (2005) Constructing minimal ancestral recombination graphs, J. Comput. Biol., 12(2), 147-169.

9. Song,Y.S., Hein,J. (2003) Parisimonious reconstruction of sequence evolution and haplotype blocks: finding the minimum number of recombination events, Proceedings of the Third International Workshop on Algorithms in Bioinfomatics (WABI 2003), 287-302.

10. Szatkiewicz,J.P., Beane,G.L., Ding,Y., Hutchins,L., Pardo-Manuel de Villena,F., Churchill,G. (2008) An imputed genotype resource for the laboratory mouse, Mamm Genome, 19:199.

11. Ukkonen, E. (2002) Finding founder sequences from a set of recombinants, WABI 2002, 277-286.

12. Wang,L., Zhang,K., Zhang,L. (2001) Perfect phylogenetic networks with recombinaiton, J. Comput. Biol., 8, 69-78.

13. Wu, Y., Gusfield, D. (2007) Improved algorithms for inferring the minimum mosaic of a set of recombinants, CPM 200\%, 150-161.

14. Zhang,K., Deng,M., Chen,T., Waterman,M.S., Sun,F. (2003) A Dynamic programming algorithm for haplotype block partitioning, PNAS., 99, 73357339 .

15. Zhang, Q., Wang, W., McMillan, L., Villena, F.P., Threadgill, D. (2008) Inferring genome-wide mosaic structure, UNC Tech. Report (2008).

16. http://cgd.jax.org/ImputedSNPData/imputedSNPs.htm. 